\title{
Modeling the ISM Properties of Metal-Poor Galaxies and Gamma-Ray Burst Hosts
}

\author{
Emily M. Levesque ${ }^{1}$, Lisa J. Kewley ${ }^{1}$, Kirsten Larson ${ }^{1}$, \\ Leonie Snijders ${ }^{2}$ \\ ${ }^{1}$ Institute for Astronomy, University of Hawaii \\ 2680 Woodlawn Dr., Honolulu, HI 96822 \\ ${ }^{2}$ Leiden Observatory, Leiden University \\ P.O. Box 9513, 2300 RA Leiden, The Netherlands
}

\begin{abstract}
Recent research has suggested that long-duration gamma-ray bursts (LGRBs) occur preferentially in low-metallicity environments, but the exact nature of this correlation is currently a matter of intense debate. We use the newest generation of the Starburst99/Mappings code to generate an extensive suite of cutting-edge stellar population synthesis models, covering a wide range of physical parameters specifically tailored for modeling the ISM environments of metal-poor galaxies and LGRB host galaxies. With our models, we generate optical emission line diagnostics, which will allow us to examine the ISM properties and stellar populations of a variety of galaxy populations in unprecedented detail. While accurately modeling low-metallicity galaxies still poses a challenge to these models, future improvements to these grids will have profound consequences for our understanding of metal-poor galaxies, their ISM environments, and the nature of their role as the hosts of LGRBs.
\end{abstract}

Keywords. galaxies: ISM, galaxies: abundances, galaxies: stellar content

\section{Introduction}

Long-duration gamma-ray bursts (LRGBs) have long been considered excellent tools for probing star-formation in distant galaxies. In recent years, however, several studies have uncovered a connection between LGRBs and low-metallicity galaxies that could threaten their utility as unbiased tracers of star formation in our universe. Such studies find that these events' hosts lie below the standard mass-metallicity relation for dwarf galaxies (Stanek et al. 2006, Kewley et al. 2007), and are morphologically and chemically distinct from the hosts of burstless core-collapse SNe (Fruchter et al. 2006, Modjaz et al. 2008).

However, there are many authors that doubt the validity of this apparent low-metallicity bias, noting that it could be, for example, simply a by-product of a bias towards young stellar population age (Bloom et al. 2002, Berger et al. 2007). There are also arguments that such a bias should not affect the utility of LGRBs as star formation tracers at high redshift, where the mean metallicity is lower as a whole (Fynbo et al. 2006).

Understanding the relationship between LGRBs and low-metallicity environments is extremely important, as it could challenge the use of these events as tracers of star formation in normal galaxies. A metallicity bias would suggest that LGRBs are not the best means of probing early star formation, as they would be considerably less likely to occur in normal star-forming galaxies (Stanek et al. 2006).

This kind of detailed analyses of LGRB ISM environments requires the use of a comprehensive, detailed, and robust grid of stellar population synthesis models that can reproduce the observed spectra of metal-poor galaxies and LGRB hosts. With such models, the ISM properties of the galaxies can be probed in detail, and the similarities and 
differences between the two populations can be quantified and applied to our current understanding of LGRB progenitor models and metal-poor galaxy properties.

In this paper, we present an extensive suite of model galaxy spectra covering a wide range of physical parameters, generated using the newest generation of the Starburst99/ Mappings III code. We demonstrate the application of these diagnostics to spectra of a variety of galaxy populations, examine the results of these comparisons, and discuss future work in this area.

\section{Starburst99/Mappings III Model Grids}

\subsection{Model Grid Parameters}

To model our sample of galaxies we have used the Starburst99 code (Leitherer et al. 1999, Vázquez \& Leitherer 2005) to generate theoretical spectral energy distributions (SEDs), which in turn were used in Mappings III photoionization models to produce model galaxy spectra that could be compared to our observations.

Starburst99 is an evolutionary synthesis code that can be used to generate synthetic ionizing far-ultraviolet (FUV) radiation spectra as a function of metallicity, star formation history, and the age and evolution of the stellar populations. These populations are produced by use of model stellar atmospheres and spectra along with evolutionary tracks for massive stars. Starburst99 generates the final synthetic FUV spectrum as output, which is then taken as input by the Mappings III shock and photoionization code, originally developed by Binette et al. (1985) and most recently improved to include a more sophisticated treatment of dust (Groves et al. 2004). For a more detailed discussion of Mappings III see Dopita et al. (2000) and Kewley et al. (2001). The parameters taken as inputs by Starburst99 and Mappings III are illustrated in Figure 1.

With these codes we have computed a complete grid of plane-parallel isobaric photoionization models, ranging in age from 0 to $10 \mathrm{Myr}$ in increments of $0.5 \mathrm{Myr}$. When generating our Starburst99/Mappings III stellar population synthesis models, we adopted a broad grid of parameters to facilitate comparison with a wide range of galaxy samples:

Star Formation History: We model both a zero-age instantaneous burst of star formation, with a fixed mass of $10^{6} M_{\odot}$, and a continuous star formation history where the star formation rate (SFR) is constant at a rate of $1 M_{\odot}$ per year, starting from an initial time and assuming a stellar population that is large enough to render the fluctuating contributions from high-mass stars negligible.

Metallicity: We model the full range of metallicities available from the evolutionary tracks of the Geneva group, which includes five metallicities of $z=0.001,0.004,0.008$, 0.02 , and 0.04 , where $Z_{\odot}=0.02$

Evolutionary Tracks: We adopted the two evolutionary tracks of the Geneva group that are currently available in Starburst99: the Geneva "standard" (STD) mass loss tracks, and the Geneva "high" (HIGH) mass loss tracks. The STD evolutionary tracks were originally published in a series of papers by the Geneva group (Schaller et al. 1992; Schaerer et al. 1993a, 1993b; Charbonnel et al. 1993); the HIGH tracks, published in Meynet et al. (1994), include higher mass loss rates derived by doubling those adopted in the STD models. While many advances have since been made in our understanding of stellar physics, adopting these tracks in our stellar population synthesis models is still advisable. The STD mass loss tracks are the more applicable of the two when considering the effects of wind clumping on mass loss rates (Crowther et al. 2002), and the HIGH mass loss tracks produce a reasonable approximation of the mass loss rates resulting from 

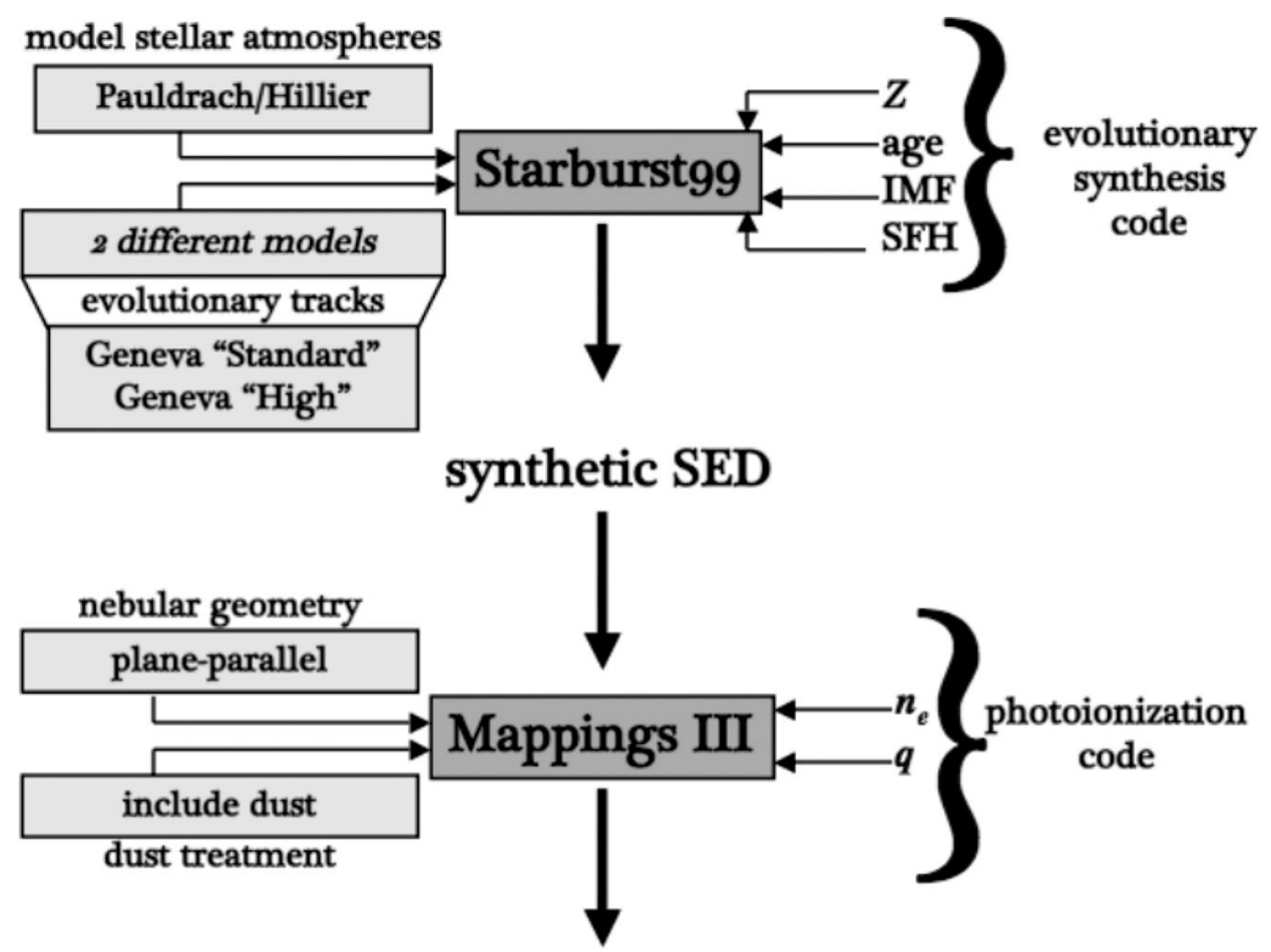

\section{model galaxy spectrum}

Figure 1. Illustration of the inputs taken by the Starburst99 and Mappings III codes when generating our model grids.

the effects of rotation, when surface mixing results in an earlier start of the WR phase (Meynet, private communication).

Ionization parameter: In Mappings III, the ionization parameter $q\left(\mathrm{~cm} \mathrm{~s}^{-1}\right)$ is defined as the maximum velocity possible for an ionization front being driven by the local radiation field, and can be translated to a dimensionless ionization parameter by dividing by the speed of light (Dopita et al. 2000). In our model grid, we adopted seven different ionization parameters $\left(q=1 \times 10^{7}, 2 \times 10^{7}, 4 \times 10^{7}, 8 \times 10^{7}, 1 \times 10^{8}, 2 \times 10^{8}\right.$, and $\left.4 \times 10^{8} \mathrm{~cm} / \mathrm{s}\right)$.

Electron density: We adopt two different electron densities $n_{e}$ for this work, of $n_{e}=10$ and $n_{e}=100$. We assume an isobaric density structure for these models, and thus $n_{e}$ is specified by the dimensionless pressure/mean temperature ratio. For the remainder of this paper, we present results that adopt $n_{e}=100$.

\section{Comparison to Observations}

\subsection{Galaxy Samples}

With the generation of these grids complete, we can now compare the results of our Starburst99/Mappings models to observed spectra from a variety of galaxy populations:

Metal-Poor Galaxies (MPGs): The MPG spectra were selected by Brown et al. (2008) from a larger survey of blue compact galaxies (BCGs). The galaxies in this sample are all categorized as MPGs: their metallicities, $12+\log (\mathrm{O} / \mathrm{H})$, range from 7.41 to 8.32 as 

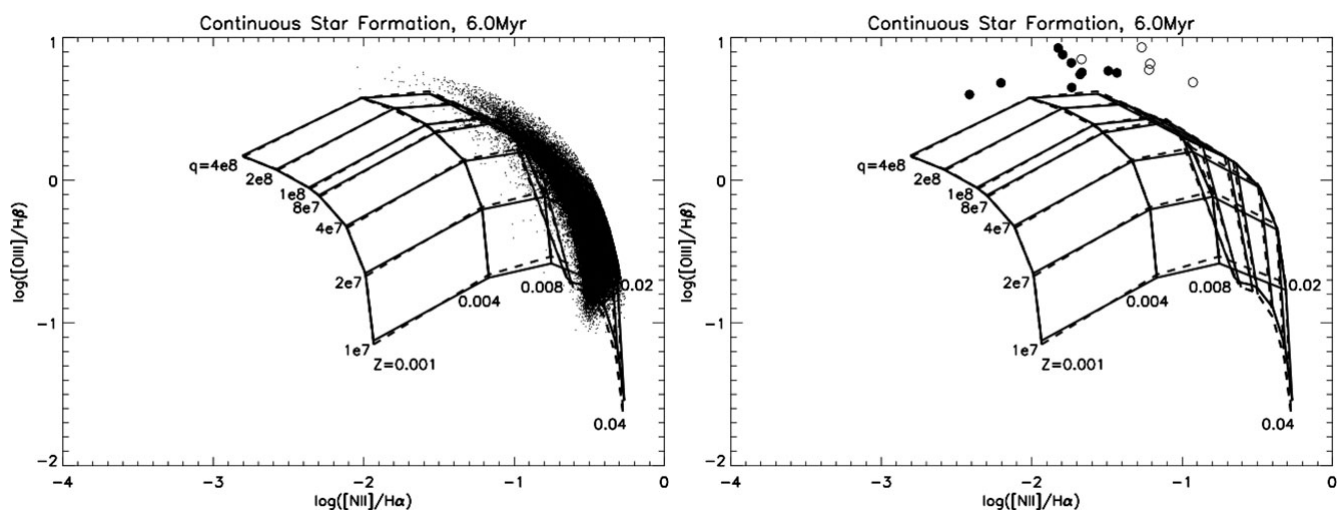

Figure 2. $[\mathrm{NII}] / \mathrm{H} \alpha$ vs. $[\mathrm{OIII}] / \mathrm{H} \beta$ emission line diagnostic grids for a $6.0 \mathrm{Myr}$ continuous star formation model grid; an electron density of 100 is assumed. Left: the grid is in excellent agreement with the position of 60,920 SDSS star-forming galaxies (small points). Right: the agreement of the grid with the MPGs (filled circles), and LGRB hosts (open circles). We see that the models are in poor agreement with the MPG and LGRB host samples. In the electronic color version of these figures, lines of constant metallicity are shown in blue/green, while lines of constant ionization parameter are shown in red/yellow.

determined by the electron temperature $\left(T_{e}\right)$ diagnostic, and from 7.66 to 8.25 using the $\mathrm{R}_{23}$ diagnostic put forth by Kewley \& Dopita (2002) and refined by Kobulnicky \& Kewley (2004). These galaxies inhabit the same region of the luminosity-metallicity plot for dwarf irregular galaxies as LGRB hosts, showing that both of these populations have distinctly low metallicites relative to their luminosities (for more information see Figure 6 of Brown et al. 2008, and discussion therein).

$L G R B$ Host Galaxies: In addition to the metal-poor galaxies, we include a sample of galaxies that have hosted LGRBs, taken from the Gamma-ray burst Host Studies (GHostS) archive (Savaglio et al. 2006). We have selected a small sample of LGRB host galaxies that have accurate spectroscopic observations with sufficient spectral coverage and emission line detections.

Sloan Digital Sky Survey (SDSS) galaxies: Finally, to compare our models to the general local $(z \leqslant 0.3)$ galaxy population, we plot a sample of 60,920 star forming galaxies from SDSS; for more discussion of this sample see Kewley \& Ellison (2008).

The MPG and SDSS galaxy fluxes were corrected for local extinction effects based on the $\mathrm{H} \alpha / \mathrm{H} \beta$ emission line ratio, assuming the Balmer decrement for case $\mathrm{B}$ recombination $\left(\mathrm{H} \alpha / \mathrm{H} \beta=2.85\right.$ for $\mathrm{T}=10^{4} \mathrm{~K}$ and $n_{e} \sim 10^{2}-10^{4} \mathrm{~cm}^{-3}$, following Osterbrock 1989) and the Cardelli et al. (1989) reddening law with the standard total-to-selective extinction ratio $R_{V}=3.1$ (the line fluxes for the LGRB host galaxies were taken from the literature and had been previously dereddened).

\subsection{Emission Line Diagnostics Grids}

For our analyses, we have plotted the models in a series of emission line diagnostic grids, comparing a variety of line ratios selected to examine the evolution of specific properties such as metallicity and ionization parameter across the full parameter space of our models. In Figure 2, we compare the HIGH (solid line) and STD (dotted line) model grids to the position of these galaxy samples.

It is clear from examining these figures that, while the models agree with the position of the SDSS galaxies (Figure 2, left panel), they are in very poor agreement with the MPG and LGRB host samples (Figure 2, right panel). We conclude from our comparison 
of these observations with our model grid that, while the models can be applied quite effectively to the general galaxy population, further improvements are needed before they can be accurately used to determine the ISM properties of low-metallicity galaxy populations.

\section{Discussion and Future Work}

One potential means of improving our models concerns the new generation of the Geneva evolutionary tracks, which accommodate for the first time the effects of rotation on the stellar population (Vázquez et al. 2007). The effects of rotation are particularly critical at lower metallicities. Expanding our current grid of evolutionary models by include the rotating evolutionary tracks as they become available will help us to further probe the effect that these tracks have on the eventual outcome of the models, as well as take important strides towards improving these grids and making them applicable to lower-metallicity galaxy populations.

\section{References}

Berger, E., Fox, D. B., Kulkarni, S. R., Frail, D. A., \& Djorgovski, S. G. 2007 ApJ, 660, 504

Binette, L., Dopita, M. A., \& Tuohy, I. R. 1985 ApJ, 297, 476

Bloom, J. S., Kulkarni, S. R., \& Djorgovski, S. G. 2002, ApJ, 121, 1111

Brown, W., Kewley, L. J., \& Geller, M. J. 2008, AJ, 135, 92

Cardelli, J. A., Clayton, G. C., \& Mathis, J. S. 1989, ApJ, 345, 245

Charbonnel, C., Meynet, G., Maeder, A., Schaller, G., \& Schaerer, D. 1993, A\&SAS, 101, 415

Crowther, P. A., Dessart, K., Hillier, D. J., Abbott, D. B., \& Fullterton, A. W. 2002, A\&\&A, 392, 653

Dopita, M. A., Kewley, L. J., Heisler, C. A., \& Sutherland, R. S. 2000, ApJ, 542, 224

Fruchter, A .S. et al. 2006, Nature, 441, 463

Fynbo, J. P. U., et al. 2006, A\&SA, 451, 47

Groves, B., Dopita, M., \& Sutherland, R. 2004, ApJS, 153, 9

Kewley, L. J., Brown, W. R., Geller, M. J., Kenyon, S. J., \& Kurtz, M. J. 2007, AJ, 133, 882

Kewley, L. J. \& Dopita, M. A. 2002, ApJS, 142, 35

Kewley, L. J., Dopita, M. A., Sutherland, R. S., Heisler, C. A., \& Trevena, J. 2001, ApJ, 556, 121

Kewley, L. J., \& Ellison, S. 2008, arXiv:0801.1849

Kewley, L. J., Groves, B., Kauffman, G., \& Heckman, T. 2006, MNRAS, 372, 961

Kobulnicky, H. A. \& Kewley, L. J. 2004, ApJ, 617, 240

Kong, X. \& Cheng, F. Z. 2002, A\&A A, 389, 845

Leitherer, C., et al. 1999, ApJS, 123, 3

Meynet, G., Maeder, A., Schaller, G., Schaerer, D., \& Charbonnel, C. 1994, A\&AS, 103, 97

Modjaz, M., Kewley, L. J., Kirshner, R. P., Stanek, K. Z., Challis, P., Garnavich, P. M., Greene, J. E., Kelly, P. L., Prieto, J. L. 2008, AJ 135, 1136

Osterbrock, D. 1989, Astrophysics of gaseous nebulae and active galactic nuclei (University Science Books)

Savaglio, S., Glazebrook, K., \& Le Borgne, D. 2006, in: S. S. Holt, N. Gehrels, \& J. A. Nousek (eds.), American Institute of Physics Conference Series (Publ. de l'Observatoire de Paris), p. $540-545$

Schaerer, D., Charbonnel, C., Meynet, G., Maeder, A., \& Schaller, G. 1993a, A\& $A S, 102,339$

Schaerer, D., Meynet, G., Maeder, A., \& Schaller, G. 1993b, A\&AS, 98, 523

Schaller, G., Schaerer, D., Meynet, G., Maeder, A. 1992, A\&BAS, 96, 269

Stanek, K. Z., et al. 2006, Acta Astron., 56, 333

Vázquez, G. A. \& Leitherer, C. 2005, ApJ, 621, 695

Vázquez, G. A., Leitherer, C., Schaerer, D., Meynet, G., \& Maeder, A. 2007, ApJ, 663, 995 